\title{
Bile Cast Nephropathy Secondary to Acute Transient Liver Injury and Autoimmune Haemolytic Anaemia: Case Presentation and Review of Literatures
}

\author{
Fakhriya J Alalawi ${ }^{1 *}$, Hind H AINour ${ }^{1}$, Michael Jansen² Amna K Al-Hadari ${ }^{3}$ and Mohammed Raiey ${ }^{3}$ \\ ${ }^{1}$ Specialist Senior Nephrologist, Department of Nephrology, Dubai hospital, Dubai Health Authority, Dubai, UAE \\ ${ }^{2}$ Consultant Pathologist, Department of Nephrology, Dubai hospital, Dubai Health Authority, Dubai, UAE \\ ${ }^{3}$ Consultant Nephrologist, Department of Nephrology, Dubai hospital, Dubai Health Authority, Dubai, UAE
}

\begin{abstract}
Bile cast nephropathy, or cholaemic nephrosis is a rare cause of acute renal injury with only a few cases that have been reported.

In this article, we present a patient with acute transient liver injury, autoimmune haemolytic anaemia and oligouric acute kidney injury. Kidney biopsy had confirmed presence of bile casts with evidence of acute tubular injury.
\end{abstract}

Keywords: Bilirubin; Bile cast; Bile acids; Tubulopathy; AKI: Acute kidney injury; ATN: Acute tubular necrosis; Intravascular haemolysis; HUS: Haemolytic uremic syndrome

\section{Introduction}

Bile cast nephropathy, or cholaemic nephrosis is a rare and infrequently reported cause of acute renal injury.

A case of acute bile cast nephropathy is reported here with review of the literatures.

\section{Case Report}

A 61 year old lady, with no previous co-morbidities or significant history of prior illness, had presented to the emergency unit with a history of headache, vomiting, diarrhea and fever of five days duration.

The above mentioned patient had recently travelled to Iraq and returned the day prior to admission. While in Iraq she noticed yellowish discoloration of her sclera and skin associated with headache, vomiting and reduced urine output, that all started a day after an onset of high grade fever. In Iraq her laboratory tests revealed an increase in serum bilirubin level $(7 \mathrm{mg} / \mathrm{dl})$, with an increase in liver enzymes and anemia (hemoglobin $7 \mathrm{~g} / \mathrm{dl}$ ). Few days later she developed diarrhea. There was no history of skin rash, ingestion of herbal or NSAIDS medication and no history of blood transfusion.

Upon arrival to our Emergency Room her clinical examination revealed a $\mathrm{BP}$ of $101 / 50 \mathrm{mmHg}$, pulse $82 \mathrm{~b} / \mathrm{min}$, temperature $37^{\circ} \mathrm{C}$. The patient exhibited pallor and mild jaundice. No further clinical findings were noted.

Her laboratory tests at presentation revealed: serum creatinine level of $7.3 \mathrm{mg} / \mathrm{dl}$ ( 3 days earlier in Iraq, her serum creatinine was 3.56 $\mathrm{mg} / \mathrm{dl})$, urea $163 \mathrm{mg} / \mathrm{dl}$.

Her hemoglobin level was $5.2 \mathrm{~g} / \mathrm{dl}$; white cells count $18.2 \times 10^{3} / \mu \mathrm{l}$ and platelets $123 \times 10^{3} / \mu \mathrm{l}$ (Figure 1 ).

This patient was admitted under nephrology care, started on careful hydration, and transfused with red blood cells (RBC). Antibiotics were added empirically in view of her fever and leukocytosis, however, in the ensuing days her serum creatinine had increased further to 12 $\mathrm{mg} / \mathrm{dl}$ and blood urea to $271 \mathrm{mg} / \mathrm{dl}$. Moreover she became oligouric; necessitating urgent initiation of hemodialysis.

Regarding her anemia; her hemoglobin was $5.2 \mathrm{~g} / \mathrm{dl}$ on arrival (compared to $7 \mathrm{~g} / \mathrm{dl} 3$ days earlier in Iraq, though no blood transfusion were given in Iraq), she was transfused several units of blood as packed $\mathrm{RBC}$, yet her $\mathrm{Hb}$ level dropped rapidly, with reticulocyte count of $5.59 \%$, lactic dehydrogenase (LDH) of 1771u/l (n 240-480), Direct coombs test was positive, indirect test was negative, Uric acid was 11.9 $\mathrm{mg} / \mathrm{dl}$, whereas, her coagulation profile were normal.

Blood film showed frequent schistocytes, with bitten, irregular contracted cells and polychromasia along with reduced platelets consistent with microangiopathic hemolytic anemia. Additionally Leukocytosis with left neutrophiles shift and toxic granulation was noted. Hematology consultation was pursued and a diagnosis of acute autoimmune hemolytic anemia was suggested. Patient was initiated on prednisolone $1 \mathrm{mg} / \mathrm{kg} /$ day.

The patients total bilirubin at the time of presentation was 1.3 $\mathrm{mg} / \mathrm{dl}$ (compared to $7 \mathrm{mg} / \mathrm{dl}$ only 4 days back in Iraq), Alanine transaminase (ALT/SGPT) upon admission was $53 \mu / \mathrm{l}$ (N. 0-32), aspartate aminotransferase (AST/SGOT) of $24 \mu / 1$ (N. 0-32), Gamma glutamyl transpeptidase (GGT) $60 \mu / 1$ (N.5-36), liver function test had normalized within few days of admission and serum bilirubin had declined to $0.6 \mathrm{mg} / \mathrm{dl}$.

CXR and KUB (Kidneys, ureters, and bladder) X-ray were normal, however there was an incidental finding of well-defined rounded calcified shadow noted in the right hypochondrial area suggesting a calcified hydatid cyst, CT KUB revealed presence of normal size and positions of both kidneys with no evidence of obstruction.

In summary; this 61 year old woman presented with an acute kidney injury associated with an acute autoimmune haemolytic and

*Corresponding author: Fakhriya J Alalawi, Specialist Senior Nephrologist Department of Nephrology, Dubai hospital, Dubai Health Authority, UAE, Tel: 00971509787597/0097402195000; E-mail: fjalalawi08@yahoo.co.uk/fjalalawi@ dha.gov.ae

Received: April 27, 2015; Accepted: July 09, 2015; Published: July 15, 2015

Citation: Alalawi FJ, AINour HH, Jansen M, Al-Hadari AK, Raiey M (2015) Bile Cast Nephropathy Secondary to Acute Transient Liver Injury and Autoimmune Haemolytic Anaemia: Case Presentation and Review of Literatures. J Nephrol Ther 5: 206. doi:10.4172/2161-0959.1000206

Copyright: (c) 2015 Alalawi FJ, et al. This is an open-access article distributed under the terms of the Creative Commons Attribution License, which permits unrestricted use, distribution, and reproduction in any medium, provided the original author and source are credited. 
Citation: Alalawi FJ, AINour HH, Jansen M, Al-Hadari AK, Raiey M (2015) Bile Cast Nephropathy Secondary to Acute Transient Liver Injury and Autoimmune Haemolytic Anaemia: Case Presentation and Review of Literatures. J Nephrol Ther 5: 206. doi:10.4172/2161-0959.1000206

Page 2 of 4

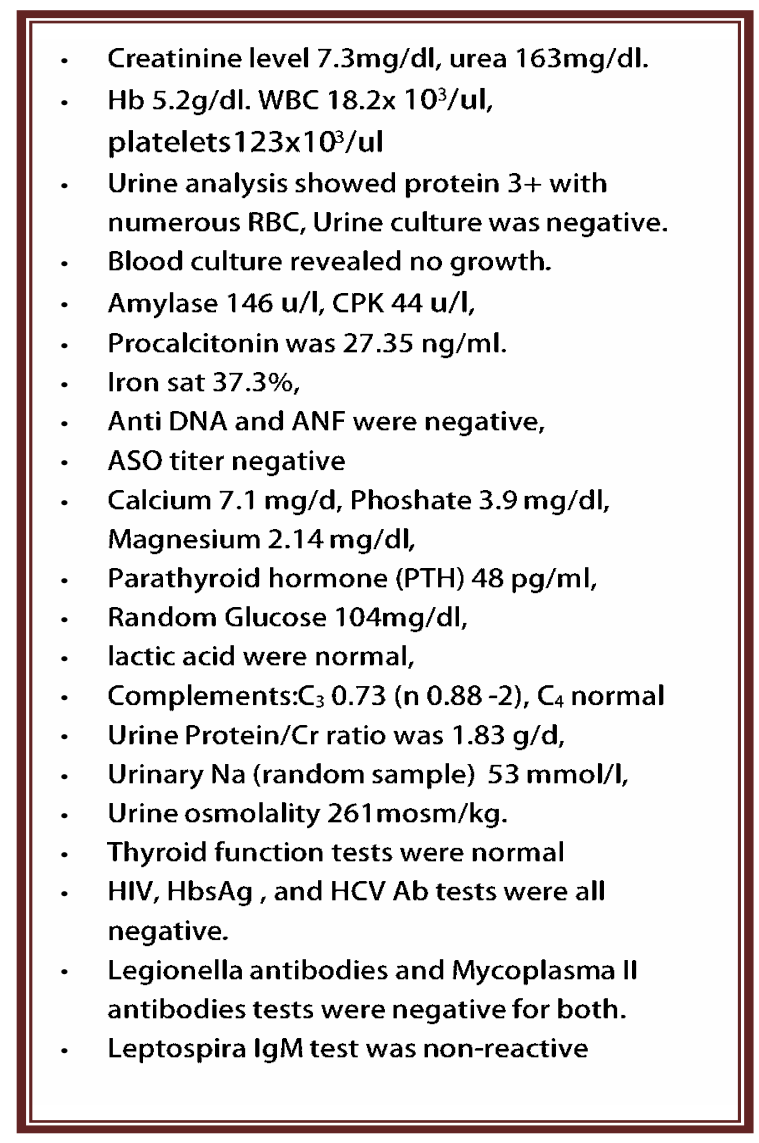

Figure 1: Lab tests at presentation.

evidence of resolving acute liver injury. We considered the differential diagnosis to include acute HUS/TTP which was high on the list, although, positive direct coombs test was made this possibility unlikely; other differentials includes post infectious glomerulonephritis or sepsis with ATN and acute liver injury, though the cause was uncertain, a possible viral etiology were postulated for all. To confirm the diagnosis, she underwent an ultrasound guided kidney biopsy. The biopsy showed a total of twenty seven glomeruli were present in the examined planes of section. No crescents were present, no sclerosed glomeruli were identified. Although some glomeruli appeared subjectively swollen, two showed mildly accentuated lobularity and one showed segmental endocapillary proliferation. The capillary loop contours were normal and the capillary lumens were patent. No intravascular thrombi were seen. The small vessels show mild arteriosclerosis, while the larger vessels are within normal limits with no evidence of vasculitis (Figures 2-4).

The tubules showed a variety of appearances - while half were normal; the remaining tubules were abnormal; these were dilated and contained orange-tan pigment, and possibly red blood corpuscles. This pigment was light yellow on trichrome and light green on Fouchets, keeping with bilirubin pigment. Additionally light parenchymal staining was present on Perls stains, suggesting the presence of haemosiderin. Tubular epithelium was degenerated in areas evidenced by detachment with loss of nuclear staining intensity and regenerative features with cytoplasmic and nuclear enlargement were also present.

The interstitium showed a minimal increase in interstitial fibrosis as well as a mild to moderate increase in inflammatory cells composed predominantly of lymphocytes with occasional plasma cells and neutrophils.

Immunofluorescences staining were all negative, as in Table 1.

These findings were interpreted as consistent with resolving acute tubular necrosis involving $30 \%$ of sampled tissue with intratubular pigmented bilirubin casts. Patient underwent seven sessions of hemodialysis and had made an excellent recovery of her kidney function. Her hemoglobin level had stabilized at $8.9 \mathrm{~g} / \mathrm{dl}$ after starting steroids, while her reticulocyte count and LDH had normalized $(0.86 \%$ and $539 \mu / \mathrm{l}$ respectively) as well as uric acid $(5.8 \mathrm{mg} / \mathrm{dl})$, her urinary protein/creatinine ratio had declined to $0.2 \mathrm{~g} / \mathrm{dl}$.

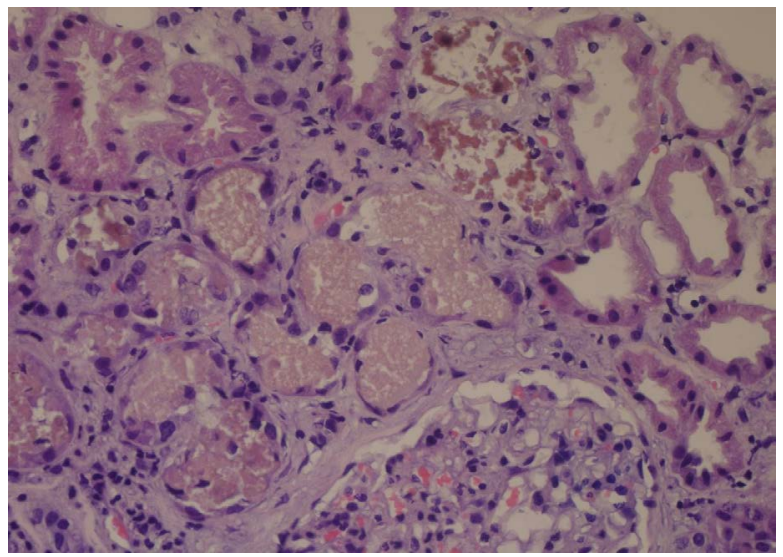

Figure 2: HE stain 200X magnification - Renal tissue including a glomerulus and tubules; the central tubules contain orange tan pigmented material surrounded by tubular epithelium showing regenerative changes. Adjacent tubules to the top left and right show effete red cells.

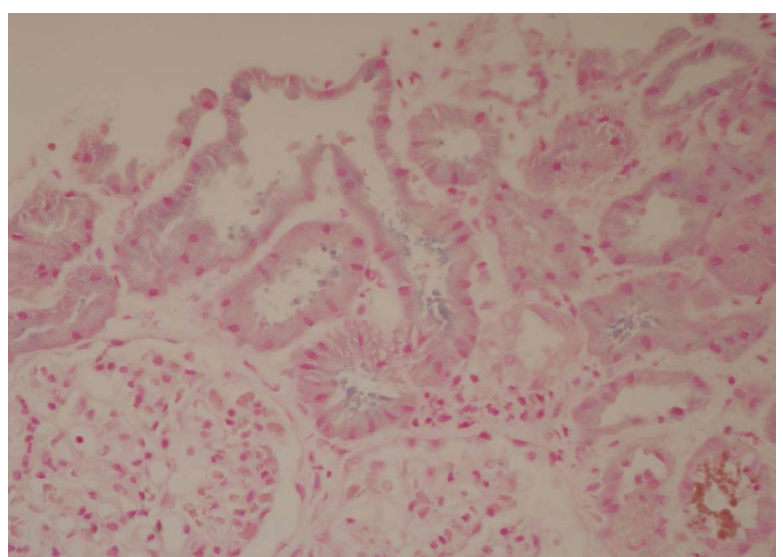

Figure 3: Perls' stain 200X magnification - Some of the material shows light blue staining indicating a positive reaction for iron in haemosiderin pigment; adjacent tubules however show intraluminal material that is Perls' negative.

\begin{tabular}{|c|c|}
\hline Antibody & Score \\
\hline $\operatorname{lgM}$ & Nil \\
\hline $\lg G$ & Nil \\
\hline $\lg \mathrm{A}$ & $\mathrm{Nil}$ \\
\hline C3 & Nil \\
\hline C1q & Nil \\
\hline Fibrin & Nil \\
\hline
\end{tabular}

Table 1: Immunofluorescences staining. 


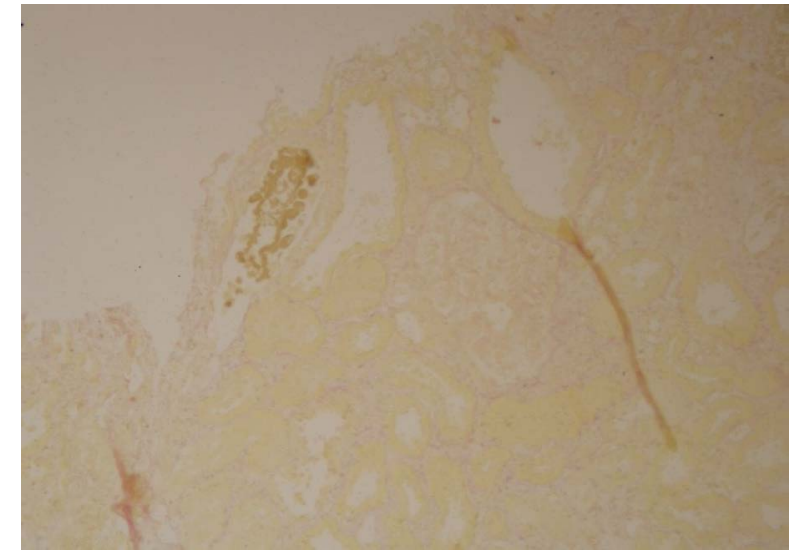

Figure 4: Fouchet stain 200X magnification - The material in the tubules stains light green in keeping with bile pigment

After discharge this patient continued to remain stable; six months later she remained asymptomatic, her biochemical profile showed a urea of $68 \mathrm{mg} / \mathrm{dl}$ and creatinine of $1.1 \mathrm{mg} / \mathrm{dl}$ while her $\mathrm{Hb}$ was $10.2 \mathrm{~g} / \mathrm{dl}$.

\section{Discussion}

Bile cast nephropathy, is a rare cause of acute renal injury. The term cholemic nephrosis had been used previously to describe functional and histologic abnormalities in the kidneys of jaundiced patients [13] however; this term has disappeared altogether from the nephrology textbooks. A major drawback to the interpretation of the original data on cholemic nephrosis is that they were obtained by autopsy of jaundiced patients, who died from a variety of causes that may have led to acute tubular necrosis $[1,3]$. The current term used frequently in the literatures is bile cast nephropathy or jaundice-related tubulopathy.

The association of jaundice and acute kidney injury is not uncommon; yet very few cases of acute bile cast nephropathy have been reported in conjunction with acute rather than chronic hepatic injury.

The hallmark of bile cast nephropathy is essentially a tubulopathy, with a spectrum that can range from mild proximal tubulopathy to an acute tubular necrosis [1-4].

The exact pathophysiological mechanism of bile cast nephropathy remains uncertain despite a number of experimental studies in this field. It is thought that hyperbilirubinaemia might cause kidney damage in two ways: bile casts causing tubular obstruction (as possibly seen in our patient) leading to acute tubular injury in similar fashion to myeloma cast or rhabdomyolysis (bile cast nephropathy) or alternately due to bilirubin exerting a direct nephrotoxic effect on renal tubular epithelium with subsequent impairment of tubular function especially the function of proximal convoluted tubule (proximal tubulopathy) [1, 3-5]. Both mechanisms could account for our patient's presentation.

The tubular nephrotoxic effect could largely be triggered by oxidative stress insults to the tubular cells and mitochondrial membrane as demonstrated in rat's models of acute bile duct ligation $[2,3]$, additionally, sulfated bile salts can influence renal function by $\mathrm{Na}-\mathrm{K}$ anti-port inhibition in the brush border of the tubular cells; hence the urinary-excreted toxic bile acids represent a pivotal trigger for renal tubular epithelial injury leading to bile cast nephropathy $[1-3,6]$.

Bile nephrotoxicity to the renal tubular cells can vary from tubular injury-induced electrolyte impairment e.g. hyponatremia, hypokalemia, hypouricemia, normal gap metabolic acidosis, and Fanconi syndrome, to acute tubular necrosis, that could be associated with a tubulointerstitial nephritis and periglomerular fibrosis [1,2]. Interestingly few studies had noted glomerular changes in addition to tubular damage that was eventually called a "nebulous entity" [7,8], our patient showed some nonspecific glomerular changes.

Hyponatremia (as in our case) is present in more than $70 \%$ of reversible obstructive jaundiced patients. Moreover, bilirubin inhibits uric acid absorption at S1 and S3 segments of the proximal tubule, causing hyperuricosuria [4] and profound hypouricemia [3].

Contrary to proximal tubulopathy, Bilirubin casts are deposited mainly within distal renal tubules attributed to relative insolubility of bile acids combined with the low $\mathrm{pH}$ environment of the distal nephrons, though casts can be in the proximal tubules as well. Hypertrophy of the tubular cells is observed in patients with bile cast nephropathy [5].

The extent of intra-renal bile cast accumulation correlates with the severity of liver injury as measured by serum total bilirubin and alkaline phosphatase, similarly, the amount of bile casts at biopsies correlates with the severity of acute tubular injury [1].

Low serum albumin concentration and/or endotoxemia, could be a risk factors for development of renal failure in bile cast nephropathy $[1,3]$. Endotoxemia has been attributed to the reduced hepatic clearance of endotoxin from the portal circulation by dysfunctional Kuppfer cells, and to the lack of endotoxin binding in the gut by bile acids [3]. Endotoxemia can cause a pro-inflammatory cytokine response, thereby reducing serum albumin concentrations, which in turn could increase the unbound fraction of bilirubin and bile acids, thereby increasing the filtered amount of these potential cytotoxic substances in to the intratubular lumen $[3,8]$. Usually these changes are observable if the total serum bilirubin concentration exceeds $21 \mathrm{mg} / \mathrm{dL}$ [3].

Bile cast nephropathy can be suspected clinically when extreme hyperbilirubinaemia and acute kidney injury coincide. Although it may be confirmed by the observing of bile casts in a kidney biopsy as in this case; the diagnosis can also be strongly suggested by examining urine samples for bile crystals although this is admittedly rare [5].

In our patient, liver damage (as evaluated by biochemistry) was transient suggesting another factor augmented the precipitation of bile cast in renal tubules. We attributed this to the acute hemolytic anemia. We suggested this in view of the biopsy findings of degenerate red blood corpuscles along with the pigmented bile casts. We note however that bile cast is usually absent in jaundice secondary to hemolysis as bilirubin here is of indirect or unconjugated type, and the renal casts identified in biopsies of patients with hemolysis related kidney injury are primarily of hemosiderin pigment within proximal tubular epithelial cells (hemosiderosis), and hemoglobin obstructive casts in distal nephron segments $[1,9,10]$. Scanty haemosiderin material was present in our biopsy consistent with a minor contribution from such.

Bile cast nephropathy is treated by treating the liver injury. Recovery of renal function may take several weeks depending on the extent of proximal tubulopathy and bile cast formation [3,11]. Only in those patients with profound jaundice (when the total plasma bilirubin concentration exceeds $20 \mathrm{mg} / \mathrm{dL}$ ), a moderate reduction in the GFR with increased natriuresis were observed [3].

\section{Conclusion}

We present here an unusual case of acute kidney injury secondary 
Citation: Alalawi FJ, AINour HH, Jansen M, Al-Hadari AK, Raiey M (2015) Bile Cast Nephropathy Secondary to Acute Transient Liver Injury and Autoimmune Haemolytic Anaemia: Case Presentation and Review of Literatures. J Nephrol Ther 5: 206. doi:10.4172/2161-0959.1000206

to bile cast nephropathy occurring in a context of acute and transient liver injury with a concomitant autoimmune hemolysis, although the cause was uncertain, possible viral etiology were postulated for all. Patient had made a remarkable recovery of kidney function.

\section{References}

1. van Slambrouck CM, Salem F, Meehan SM, Chang A (2013) Bile cast nephropathy is a common pathologic finding for kidney injury associated with severe liver dysfunction. Kidney Int 84: 192-197.

2. Fickert $\mathrm{P}$, Krones $\mathrm{E}$, Pollheimer MJ, Thueringer A, Moustafa T, et al. (2013) Bile Acids Trigger Cholemic Nephropathy in Common Bile-Duct-Ligated Mice. Hepatology 58: 2056-2069.

3. Betjes MG, Bajema I (2006) The pathology of jaundice-related renal insufficiency: cholemic nephrosis revisited. J Nephrol 19: 229-233.

4. Tiranathanagul K, Leelahavanichkul A, Eiam-Ong S (2012) Hyperbilirubinemiaassociated Renal Disorders; In: Jakub F. Novotny and Florian Sedlacek (edt) Bilirubin: Chemistry, Regulation and Disorder, Nova Science Publishers, New York, USA.
5. van der Wijngaart $\mathrm{H}$, van Dam B, van den Berg JG, Krul-Poel YH, Klemt-Kropp $M$, et al. (2014) A 73-year-old male with jaundice and acute kidney injury. Neth J Med 72: 95-99.

6. Sellinger M, Haak K, Burckhardt G, Gerok W, Knauf H (1990) Sulfated bile acids inhibit $\mathrm{Na}(+)-\mathrm{H}+$ antiport in human kidney brush-border membrane vesicles. Am J Physiol 258: F986-F991.

7. Sant SM, Purandare NM (1965) Cholemic nephrosis - an autopsy and experimental study. J Postgrad Med 11: 79-89.

8. Odell GB (1966) The distribution of bilirubin between albumin and mitochondria J Pediatr 68: 165-180.

9. Khalighi MA, Henriksen KJ, Chang A, Meehan SM (2015) Intratubular Hemoglobin Casts in Hemolysis-Associated Acute Kidney Injury. Am J Kidney Dis 65: $337-341$.

10. Zager RA, Gamelin LM (1989) Pathogenetic mechanisms in experimental hemoglobinuric acute renal failure. Am J Physiol 256: F446-F455.

11. Sequeira A, Gu X (2015) Bile cast nephropathy: An often forgotten diagnosis. Hemodialysis International 19: 132-135. 